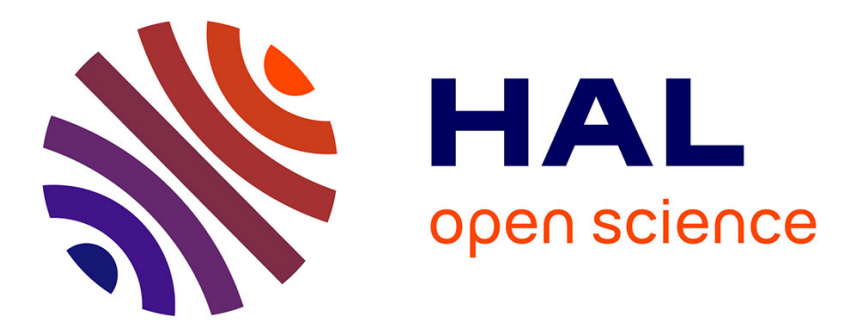

\title{
Blow-up and global existence for a kinetic equation of swarm formation
}

Miroslaw Lachowicz, Henryk Leszczyński, Martin Parisot

\section{To cite this version:}

Miroslaw Lachowicz, Henryk Leszczyński, Martin Parisot. Blow-up and global existence for a kinetic equation of swarm formation. Mathematical Models and Methods in Applied Sciences, 2017, 27 (6), pp.22. 10.1142/S0218202517400115 . hal-01370006

\section{HAL Id: hal-01370006 \\ https://inria.hal.science/hal-01370006}

Submitted on 26 Sep 2016

HAL is a multi-disciplinary open access archive for the deposit and dissemination of scientific research documents, whether they are published or not. The documents may come from teaching and research institutions in France or abroad, or from public or private research centers.
L'archive ouverte pluridisciplinaire HAL, est destinée au dépôt et à la diffusion de documents scientifiques de niveau recherche, publiés ou non, émanant des établissements d'enseignement et de recherche français ou étrangers, des laboratoires publics ou privés. 


\title{
BLOW-UP AND GLOBAL EXISTENCE FOR A KINETIC EQUATION OF SWARM FORMATION
}

\author{
MIROSŁAW LACHOWICZ *, HENRYK LESZCZYŃSKI †and MARTIN PARISOT ‡
}

September 26, 2016

\begin{abstract}
In the present paper we study possible blow-ups and global existence for a kinetic equation that describes swarm formations in the variable interacting rate case.
\end{abstract}

\section{Introduction}

In Ref. [17] a general model of swarming behavior of an individual population was proposed and studied. The main aim in that paper was to study the macroscopic (so-called "hydrodynamic") limit. The mathematical structure that was proposed seems very rich and interesting from mathematical point of view - see the analysis of its simplification in Ref. [14].

Let $f=f(t, x, v)$ be a probability density of individuals at time $t \geq 0$ at position $x \in \Omega$ and with velocity $v \in \mathbb{V} ; \Omega \subset \mathbb{R}^{d}$ and $\mathbb{V} \subset \mathbb{R}^{d}$ are domains in $\mathbb{R}^{d} ; \Omega$ is the set of positions whereas $\mathbb{V}$ is the set of velocities of individuals. We assume that $\mathbb{V}$ is a bounded domain. However the strait generalization to an unbounded domain is possible. The evolution of populations at the mesoscopic scale is defined by the nonlinear integro-differential Boltzmannlike equation, see Ref. [17],

$$
\partial_{t} f(t, x, v)+v \cdot \nabla_{x} f(t, x, v)=\frac{1}{\varepsilon} Q[f](t, x, v),
$$

${ }^{*}$ Institute of Applied Mathematics and Mechanics, Faculty of Mathematics, Informatics and Mechanics, University of Warsaw, ul. Banacha 2, 02-097 Warsaw, Poland. lachowic@mimuw.edu.pl

${ }^{\dagger}$ Institute of Mathematics, University of Gdańsk, ul. Wita Stwosza 57, 80-952 Gdańsk, Poland. hleszcz@mat.ug.edu.pl

${ }^{\ddagger}$ INRIA Paris, ANGE Project-Team, 75589 Paris Cedex 12, France; CEREMA, ANGE Project-Team, F-60280 Margny-Lès-Compiègne, France; LJLL, UPMC Université Paris VI, Sorbonne Universités, UMR CNRS 7958, F-75005 Paris, France. martin.parisot@inria.fr 
where

$$
\begin{aligned}
& Q[f](t, x, v)=\int_{\mathbb{V}}(T[f(t, x, .)](w, v) f(t, x, w) \\
& -T[f(t, x, .)](v, w) f(t, x, v)) \mathrm{d} w .
\end{aligned}
$$

Initial data are $f(0, x, v)=f_{0}(x, v)$. The parameter $\varepsilon$ corresponds to the Knudsen number and the macroscopic limit is defined by $\varepsilon \rightarrow 0$. The nonlinear operator $Q$ describes interactions between individuals. The turning rate $T[f](v, w)$ measures the probability for an individual with velocity $v$ to change velocity into $w$. A simpler equation, with two possible velocities only, was studied in Ref. [3] — see also Ref. [4].

A similar space-independent general structure was a starting point in Refs. [10] and [12]. These papers consider an integro-differential equation for the evolution of a distribution function on the circle referred to an orientational aggregation process (see also references therein). Ref. [10] provides a bifurcation analysis of the corresponding stationary problem, whereas Ref. [12] analyzes the time-dependent problem and prove that the type of alignment depends on initial data, the law of interaction and the preferred optimal orientation.

Ref. [5] deals with modeling and simulation of swarms where interactions at the microscopic scale are modeled by stochastic games. Ref. [8] reviews the state-of-the-art of swarming at the individual-based level and the macroscopic level. Special Issue Ref. [18] is dedicated to various examples of description of collective behavior. Ref. [1] develops a modeling approach of ensembles of social agents as behavioral, evolutionary, complex systems referring to the complexity features of living systems. The microscopic approach based on stochastic processes is proposed in Refs. [7], [13], [6]. Ref. [9] deals with local stability of Dirac masses for a kinetic model of alignment.

In Ref. [17] the following general nonlinear case

$$
T[f(t, x, .)](v, w)=\sigma_{\rho, x} \beta_{\rho, x}(v, w) f(t, x, w)^{\gamma_{\rho, x}}
$$

has been proposed, where $\rho(t, x)=\int_{\mathbb{V}} f(t, x, v) \mathrm{d} v$ is the macroscopic density of individuals.

Given any $\rho$ and at any $x$, the interaction rate $\beta_{\rho, x}: \mathbb{V}^{2} \rightarrow \mathbb{R}_{+}$, the attractiveness coefficient $\gamma_{\rho, x} \in \mathbb{R}_{+}$, and $\sigma_{\rho, x}= \pm 1$ characterize the interaction between individual agents. The interaction rate $\beta_{\rho, x}$ corresponds to the tendency of individuals to switch to a different velocity. Ref. [17] proposed results of global existence in the space homogeneous case for any set of collision parameters $\sigma$ and $\gamma$ except the so-called positive gregarious interaction i.e. $\sigma=1$ and $\gamma>1$.

Equation (1) can be modified to include nonlocal (with respect to space 
variable) interactions

$$
\partial_{t} f(t, x, v)+v \cdot \nabla_{x} f(t, x, v)=\frac{1}{\varepsilon} Q_{N}[f](t, x, v),
$$

where

$$
\begin{aligned}
& Q_{N}[f](t, x, v)=\int_{\mathbb{V}} \int_{\Omega}(T[f(t, ., .)](y, w, x, v) f(t, y, w) \\
& -T[f(t, ., .)](x, v, y, w) f(t, x, v)) \mathrm{d} y \mathrm{~d} w .
\end{aligned}
$$

This is a kind of a nonlinear integro-differential Povzner-like equation (an averaged Boltzmann-like equation, cf. Ref. [2] and references therein).

Equations (1) and (2) are interesting new structures with very reach possible dynamics — see refs. [17] and [14] in particular cases. The main novelty with respect to the previous studies (Refs. [10], [12]) is that we consider non-linear turning rate characterized by the coefficient of attractiveness which control the behavior of the solutions.

The aim of the present paper is an analysis of various simplifications of Eqs. (1) and (2) in case of $\sigma=1$ and $\gamma>1$. In particular we study possible blow-ups and global existence in the space homogeneous case for variable interacting rates. Moreover we state the corresponding results for the nonlocal equation (2). The paper generalizes the previous approach for a simpler case of constant interaction rates in Ref. [14]. We also include here some new large time asymptotic behavior results for the constant interaction rates as well. The paper is organized as follows. Section 1.1 is devoted to some preliminaries concerning the space homogeneous version of the equation. In Section 1.2 we refer to the previous paper Ref. [14] and propose two theorems on the asymptotic behavior of solutions. In Section 2 we generalize the result of Ref. [14] into the variable interaction rates and we propose two theorems. Section 2.2 deals with blow-up properties in terms of $L^{p}$-norms. Section 3 indicates important open problems and perspectives.

\subsection{The space homogeneous case}

In this paper we focus on the space homogeneous case and the positive gregarious case, i.e. all functions and parameters are assumed to be $x-$ independent, $\sigma=1, \gamma>1$.

The main equation (1) reads

$$
\partial_{t} f(t, v)=(\beta * f) f^{\gamma}-\left(\beta * f^{\gamma}\right) f \quad \text { with } \quad f(0, v)=f_{0}(v),
$$

for any $v \in \mathbb{V}$. By $(\beta * f)$ we denote the convolution-like product in $\mathbb{V}$, i.e.

$$
(\beta * f)=\int_{\mathbb{V}} \beta(v, w) f(w) \mathrm{d} w .
$$

In addition, the $L^{p}$-norm in the velocity space $\mathbb{V}$ is denoted by $\|\cdot\|_{p}$. 
Convention 1. In the sequel we will write "for all" instead of "for almost all" having in mind possible modifications of the data and solutions on a set whose Lebesgue measure is equal to zero. These modifications do not change the essence and generality of our results.

From now on, we focus on interaction rate satisfying the following rather restrictive assumption.

Assumption 1. We assume that $\beta=\beta(v, w)$ is a function such that

$$
\beta(v, w)=\beta(w, v) \quad \text { and } \quad \beta_{-} \beta_{+} \leq \beta(v, w) \leq \beta_{+},
$$

for all $v, w \in \mathbb{V}$, where $0<\beta_{-} \leq 1$ and $0<\beta_{+}=\|\beta\|_{L^{\infty}\left(\mathbb{V}^{2}\right)}<\infty$.

Remark 1. We point out that Assumption 1 implies

$$
\beta_{-} \beta(\bar{v}, w) \leq \beta(v, w) \leq \frac{1}{\beta_{-}} \beta(\bar{v}, w),
$$

for all $v, w, \bar{v} \in \mathbb{V}$.

In Ref. [17] (Theorem 2.5) a local in time existence and uniqueness result was proved and the existence time was estimated from below. It was easy to see that any solution preserved the nonnegativity of the initial datum and the $L^{1}$-norm of the nonnegative initial datum. The question of global existence or possible blow-ups in finite time was remaining. We may point out an interesting structure of Eq. (3): in the RHS a competition between two terms is visible. The situation is somehow similar to those that are intensively studied in the PDEs — see e.g. Refs. [15], [19].

In Ref. [17] it is proved that if $\beta$ satisfies Assumption 1 and density probability $f_{0}$ is in $L^{\infty}(\mathbb{V})$, there exist $T>0$ and a unique solution of Eq. (3) in $C^{1}\left(0, T ; L^{\infty}(\mathbb{V})\right)$. The solution is a probability density and

$$
T \geq-\frac{\ln \left(1-\frac{\beta_{-}\left\|f_{0}\right\|_{\gamma}^{\gamma}}{\left\|f_{0}\right\|_{\infty}^{\gamma-1}}\right)}{(\gamma-1) \beta_{-} \beta_{+}\left\|f_{0}\right\|_{\gamma}^{\gamma}}
$$

However, this bound cannot result in the global existence even for small initial data. In fact using the strategy of Ref. [17], the solution is global if $\beta_{-}\left\|f_{0}\right\|_{\gamma}^{\gamma} \geq\left\|f_{0}\right\|_{\infty}^{\gamma-1}$, which occur only if $\beta_{-}=1$ (i.e. $\beta$ is a constant) and there exists $\mathbb{W} \subset \mathbb{V}$ such that $f_{0}(v)=\frac{1}{|\mathbb{W}|} \chi(v \in \mathbb{W})$, where $\chi($ true $)=1$ and $\chi($ false $)=0$. This particular case corresponds to the steady solution of (3), see Ref. [17] (Proposition 3). In the present paper we are interested in description of possible blow-ups as well as the sets of parameters for which the solution is global.

We rewrite Eq. (3) in the following form

$$
\partial_{t}\left(f^{1-\gamma} e^{-(\gamma-1) \int_{0}^{t}\left(\beta * f^{\gamma}\right) \mathrm{d} s}\right)=-(\gamma-1)(\beta * f) e^{-(\gamma-1) \int_{0}^{t}\left(\beta * f^{\gamma}\right) \mathrm{d} s}
$$


and by integration we obtain

$$
f(t, v)=\left(\frac{f_{0}^{\gamma-1}(v) e^{-(\gamma-1) \int_{0}^{t}\left(\beta * f^{\gamma}\right)(s, v) \mathrm{d} s}}{1-(\gamma-1) f_{0}^{\gamma-1} \int_{0}^{t}(\beta * f)(s, v) e^{-(\gamma-1) \int_{0}^{s}\left(\beta * f^{\gamma}\right)(r, v) \mathrm{d} r} \mathrm{~d} s}\right)^{\frac{1}{\gamma-1}} .
$$

\subsection{Few previous results in a simplified configuration}

The simplest case when $\beta$ is constant, say $\beta=1$, was discussed in Ref. [14]. We have

$$
\partial_{t} f=f^{\gamma}-\|f\|_{\gamma}^{\gamma} f
$$

and $z(t)=\int_{0}^{t}\|f\|_{\gamma}^{\gamma} \mathrm{d} s$ fulfills

$$
\mathrm{d}_{t} z=e^{-\gamma z} \int_{\mathbb{V}} \frac{1}{\left(f_{0}^{1-\gamma}-(\gamma-1) \int_{0}^{t} e^{-(\gamma-1) z} \mathrm{~d} r\right)^{\frac{\gamma}{\gamma-1}}} \mathrm{~d} v .
$$

This equation determines global existence or blow-up for Eq. (7). Let

$$
u(t)=\int_{0}^{t} e^{-(\gamma-1) z(s)} \mathrm{d} s .
$$

The function $u=u(t)$ is increasing and concave. Blow-up occurs for $T>0$ such that

$$
(\gamma-1)\left\|f_{0}\right\|_{\infty}^{\gamma-1} u(T)=1
$$

The ODE for $u$ reads

$$
\mathrm{d}_{t} u=e^{-(\gamma-1) z}
$$

and

$$
\begin{aligned}
& \mathrm{d}_{t}^{2} u=-(\gamma-1) e^{-(\gamma-1) z} \mathrm{~d}_{t} z= \\
& =-(\gamma-1) \mathrm{d}_{t} u\left(\mathrm{~d}_{t} u\right)^{\frac{\gamma}{\gamma-1}} \int_{\mathbb{V}} \frac{\mathrm{d} v}{\left(f_{0}^{1-\gamma}(v)-(\gamma-1) u\right)^{\frac{\gamma}{\gamma-1}}} .
\end{aligned}
$$

By integration we obtain

$$
\mathrm{d}_{t} u=\frac{1}{\left(\int_{\mathbb{V}} \frac{f_{0}(v) \mathrm{d} v}{\left(1-(\gamma-1) f_{0}^{\gamma-1}(v) u\right)^{\frac{1}{\gamma-1}}}\right)^{\gamma-1}} .
$$

Let $\mathbb{W}=\left\{v \in \mathbb{V}: f_{0}(v)=\left\|f_{0}\right\|_{\infty}\right\}$. In Ref. [14] we proved that if $0 \leq f_{0} \in L^{\infty}(\mathbb{V})$ is a probability density such that $|\mathbb{W}|>0$ then, for any 
$T>0$, there exists a unique solution $f=f(t)$ of Eq. $(7)$ in $C^{1}\left(0, T ; L^{\infty}(\mathbb{V})\right)$ and $f(t)$ is a probability density for each $t>0$.

Case $|W|=0$ is more complex (see Ref. [14]). We denote the RHS of Eq. (8) by $\Phi$, i.e.

$$
\Phi(u)=\left(\int_{\mathbb{V}} \frac{f_{0}(v)}{\left(1-(\gamma-1) f_{0}^{\gamma-1} u\right)^{\frac{1}{\gamma-1}}} \mathrm{~d} v\right)^{1-\gamma} .
$$

The function $\Phi$ is defined on $\left[0, u_{0}\left[\right.\right.$, where $u_{0}=\frac{1}{(\gamma-1)\left\|f_{0}\right\|_{\infty}^{\gamma-1}}$. Since $\Phi$ is continuous and monotone, we can extend it as follows

$$
\Phi\left(u_{0}\right):=\lim _{u \uparrow u_{0}} \Phi(u) .
$$

We have (see Ref. [14]) that if $0 \leq f_{0} \in L^{\infty}(\mathbb{V})$ and $|\mathbb{W}|=0$ then

1. If $\Phi\left(u_{0}\right)>0$, then there is a blow-up in a finite time $T>0$;

2. If $\Phi\left(u_{0}\right)=0$, then:

(a) if

$$
\int_{0}^{u_{0}} \frac{1}{\Phi(u)} \mathrm{d} u<\infty
$$

then there is a blow-up in a finite time $T>0$;

(b) if

$$
\int_{0}^{u_{0}} \frac{1}{\Phi(u)} \mathrm{d} u=\infty
$$

then for each $T>0$ there exists a unique solution on $[0, T]$.

This statement delivers a useful criterion of blow-up or global existence - see Ref. [14]. The analysis of Ref. [14] can be completed by a limit asymptotic behavior. We state a result on the limit distribution uniform in $\mathbb{W}$.

Theorem 1. Let $f_{0}$ be a probability density on $\mathbb{V}, f_{0} \in L^{\infty}(\mathbb{V})$ and $|\mathbb{W}|>0$. Then the unique solution $f$ of $E q$. (7) in $C^{1}\left(0, T ; L^{\infty}(\mathbb{V})\right)$, corresponding to the initial datum $f_{0}$, satisfies

$$
\lim _{t \rightarrow \infty} f(t, v)=\frac{1}{|\mathbb{W}|} \chi(v \in \mathbb{W}),
$$

where $\chi($ true $)=1$ and $\chi($ false $)=0$. 
Proof. By Eq. (6) we have

$$
f(t, v)=\frac{f_{0}(v) e^{-z(t)}}{\left(1-(\gamma-1) f_{0}^{\gamma-1}(v) u(t)\right)^{\frac{1}{\gamma-1}}} .
$$

Using (8) with $\mathrm{d}_{t} u=e^{-(\gamma-1) z(t)}$ we get

$$
z(t)=\ln \left(\int_{\mathbb{V}} \frac{f_{0}(w)}{\left(1-(\gamma-1) f_{0}^{\gamma-1}(w) u(t)\right)^{\frac{1}{\gamma-1}}} \mathrm{~d} w\right) .
$$

If we substitute $z(t)$ into Eq. (9) we obtain $f(t, v)$ as a function of $u(t)$ :

$$
f(t, v)=\frac{f_{0}(v)}{\left(1-(\gamma-1) f_{0}^{\gamma-1}(v) u(t)\right)^{\frac{1}{\gamma-1}} \int_{\mathbb{V}} \frac{f_{0}(w)}{\left(1-(\gamma-1) f_{0}^{\gamma-1}(w) u(t)\right)^{\frac{1}{\gamma-1}}} \mathrm{~d} w} .
$$

Now we may decompose $\mathbb{V}=\mathbb{W} \cup \mathbb{W}^{\prime}$ and note that $u(t) \rightarrow u_{0}$ as $t \rightarrow \infty$. Therefore the statement follows.

Analogously we prove

Theorem 2. Let $f_{0} \in L^{\infty}(\mathbb{V})$ be a probability density, $|\mathbb{W}|=0$ and $f$ be the unique solution of $E q$. (7) in $C^{1}\left(0, t_{*} ; L^{\infty}(\mathbb{V})\right)$, corresponding to the initial datum $f_{0}$, where $t_{*}$ is either $t_{*}=\infty$ or $t_{*}=T$ (a blow-up time).

1. If $\Phi\left(u_{0}\right)=0$ then

$$
\lim _{t \rightarrow t_{*}} f(t, v)=0 \quad \text { for } \quad v \notin \mathbb{W}
$$

2. If $\Phi\left(u_{0}\right)>0$ then

$$
\lim _{t \rightarrow t_{*}} f(t, v)=\frac{\tilde{f}_{0}(v)}{\left(1-\tilde{f}_{0}^{\gamma-1}(v)\right)^{\frac{1}{\gamma-1}} \int_{\mathbb{V}} \frac{\tilde{f}_{0}(w)}{\left(1-\tilde{f}_{0}^{\gamma-1}(w)\right)^{\frac{1}{\gamma-1}}} \mathrm{~d} w}
$$

where $\tilde{f}_{0}=\frac{f_{0}}{\left\|f_{0}\right\|_{\infty}}$.

Remark 2. If $|\mathbb{W}|=0$ and $\Phi\left(u_{0}\right)=0$ then the limit of $f(t, v)$, as $t \rightarrow t_{*}$, is a kind of Dirac-delta concentrated on $\mathbb{W}$. 
Remark 3. We can apply the technique developed in this sections to the following simplified version of Eq. (2)

$$
\begin{aligned}
& \partial_{t} f(t, x, v)+v \cdot \nabla_{x} f(t, x, v)= \\
& =f^{\gamma}(t, x, v)-f(t, x, v) \int_{\mathbb{V}} \int_{\Omega} f^{\gamma}(t, y, w) \mathrm{d} y \mathrm{~d} w
\end{aligned}
$$

with the initial condition $f(0, x, v)=f_{0}(x, v)$ where $f_{0}$ is a probability density on $\mathbb{R}^{d} \times \mathbb{V}$. We study this equation along characteristics

$$
\partial_{t} f^{\#}(t, x, v)=\left(f^{\gamma}\right)^{\#}(t, x, v)-f^{\#}(t, x, v) \int_{\mathbb{V}} \int_{\Omega} f^{\gamma}(t, y, w) \mathrm{d} y \mathrm{~d} w,
$$

where $f^{\#}=f^{\#}(t, x, v)$ is the function $f=f(t, x, v)$ considered along trajectories (see Ref. [11]). In the simplest case $\Omega=\mathbb{R}^{d}$ we have $f^{\#}(t, x, v)=$ $f(t, x+t v, v)$.

Integrating yields

$$
f^{\#}(t, x, v)=\frac{f_{0}(x, v) e^{-z(t)}}{\left(1-(\gamma-1) f_{0}(x, v) u(t)\right)^{\frac{1}{\gamma-1}}},
$$

where

$$
z(t)=\int_{0}^{t} \int_{\mathbb{V}} \int_{\Omega} f^{\gamma}(s, y, w) \mathrm{d} y \mathrm{~d} w \mathrm{~d} s, \quad u(t)=\int_{0}^{t} e^{-(\gamma-1) z(s)} d s .
$$

By standard arguments the local in time existence and uniqueness of mild solutions results hold in $X:=L^{1}\left(\mathbb{R}^{d} \times \mathbb{V}\right) \cap L^{\infty}\left(\mathbb{R}^{d} \times \mathbb{V}\right)$. Applying the methods of this section we obtain theorems analogous to Theorem 1 and Theorem 2 in Ref. [14].

In fact, if $0 \leq f_{0} \in L^{\infty}\left(\mathbb{R}^{d} \times \mathbb{V}\right)$ and $|\widetilde{\mathbb{W}}|>0$, where $\widetilde{\mathbb{W}}$ is defined like $\mathbb{W}$ on $\mathbb{R}^{d} \times \mathbb{V}$, then, for any $t>0$, there exists a unique mild solution $f=f(t)$ of Eq. (10) in $X$ and the solution is nonnegative. Moreover the case $|\mathbb{W}|=0$ leads to analogous conditions as before.

\section{Blow-up and existence results for variable inter- action rate}

\subsection{Analysis in $L^{\infty}(\mathbb{V})$ setting}

In the present section we are going to generalize the results of Ref. [14] into the variable interaction rate $\beta \not \equiv$ const. We use the same symbols as before for more general objects. Taking $\beta=1$ one obtains the previous statements. 
Define

$$
z(t, v)=\int_{0}^{t}\left(\beta * f^{\gamma}\right)(s, v) \mathrm{d} s \quad \text { and } \quad u(t, v)=\int_{0}^{t}(\beta * f)(s, v) e^{-(\gamma-1) z(s, v)} \mathrm{d} s .
$$

We will use the identities

$$
\partial_{t} z=\beta * f^{\gamma}, \quad \partial_{t} u=\beta * f .
$$

By Eq. (6) we have

$$
f(t, v)=\frac{f_{0}(v) e^{-z(t, v)}}{\left(1-(\gamma-1) f_{0}^{\gamma-1}(v) u(t, v)\right)^{\frac{1}{\gamma-1}}} .
$$

Directly by the definitions of $u$ and $z$ we have

$$
f_{0}^{\gamma-1}(w) u(t, w)=f_{0}^{\gamma-1}(v) u(t, v) \quad \text { and } \quad z(t, v)=z(t, w)
$$

for any $v, w \in \mathbb{W}$ with the set (cf. Convention 1)

$$
\begin{aligned}
& \mathbb{W}=\{w \in \mathbb{V}: \quad \beta(w, \widetilde{v}) \leq \beta(v, \widetilde{v}) \quad \text { and } \\
& \left.f_{0}^{\gamma-1}(w) \beta(w, \widetilde{v}) \geq f_{0}^{\gamma-1}(v) \beta(v, \widetilde{v}) \quad \text { for } \quad v, \widetilde{v} \in \mathbb{V}\right\} .
\end{aligned}
$$

By integration we obtain

$$
\partial_{t} z(t, v)=\int_{\mathbb{V}} \frac{\beta(v, w) f_{0}^{\gamma}(w) e^{-\gamma z(t, w)}}{\left(1-(\gamma-1) f_{0}^{\gamma-1}(w) u(t, w)\right)^{\frac{\gamma}{\gamma-1}}} \mathrm{~d} w .
$$

Furthermore

$$
\begin{aligned}
& \partial_{t} u(t, v)=(\beta * f)(t, v) e^{-(\gamma-1) z(t, v)}= \\
& =e^{-(\gamma-1) z(t, v)} \int_{\mathbb{V}} \frac{\beta(v, w) f_{0}(w) e^{-z(t, w)}}{\left(1-(\gamma-1) f_{0}^{\gamma-1}(w) u(t, w)\right)^{\frac{1}{\gamma-1}}} \mathrm{~d} w .
\end{aligned}
$$

We have

$$
\ln \partial_{t} u(t, v)=\ln ((\beta * f)(t, v))-(\gamma-1) z(t, v) .
$$

Keeping in mind Eq. (12) we see that a blow-up can occur when the denominator

$$
\mathfrak{D}(t, v)=1-(\gamma-1) f_{0}^{\gamma-1}(v) u(t, v)
$$

tends to 0 . If $\mathbb{W} \neq \emptyset$ then $\mathfrak{D}(t, v)$, for $v \in \mathbb{W}$, is the smallest. The denominator $\mathfrak{D}(t, v)$ is constant on $\mathbb{W}$. For $v \in \mathbb{V} \backslash \mathbb{W}$ and $w \in \mathbb{W}$ we have $\mathfrak{D}(t, w)<\mathfrak{D}(t, v)$. 
The set $\mathbb{W}$ has actually been defined in an optimal way to characterize the velocities $v \in \mathbb{V}$ that are susceptible to blow-up since it is the set of velocities where the denominator $\mathfrak{D}(t, v)$ of $f(t, v)$ is the smallest.

A blow-up can occur only in $\mathbb{W}$. It is also convenient to consider

$$
\mathbb{W}_{\beta}=\{w \in \mathbb{V}: \quad \beta(w, \widetilde{v}) \leq \beta(v, \widetilde{v}) \quad \text { for } \quad v, \widetilde{v} \in \mathbb{V}\},
$$

and

$$
\mathbb{W}_{0}=\left\{w \in \mathbb{W}_{\beta}: \quad f_{0}(w) \geq f_{0}(v) \quad \text { for } \quad v \in \mathbb{W}_{\beta}\right\}
$$

For $w \in \mathbb{W}$ and $v, \widetilde{v} \in \mathbb{V}$ we have $\beta(w, \widetilde{v}) \leq \beta(v, \widetilde{v})$. Thus $\mathbb{W} \subset \mathbb{W}_{\beta}$. Moreover $\beta(w, \widetilde{v})=\beta(\widetilde{w}, \widetilde{v})>0$ for $\widetilde{w} \in \mathbb{W}_{\beta}$. In addition

$$
f_{0}^{\gamma-1}(w) \beta(w, \widetilde{v}) \geq f_{0}^{\gamma-1}(\widetilde{w}) \beta(\widetilde{w}, \widetilde{v}), \quad \text { and } \quad f_{0}(w) \geq f_{0}(\widetilde{w}) .
$$

Therefore $\mathbb{W} \subset \mathbb{W}_{0}$. This yields

$$
\mathbb{W} \subset \mathbb{W}_{0} \subset \mathbb{W}_{\beta}
$$

Note that $\mathbb{W}$ is a generalization of the corresponding set considered in the previous section. If $w \in \mathbb{W}$ then the function $\beta$ treated as a function of the first variable has a global minimum at $w$ whereas $f_{0}$ has a global maximum at $w$. Thus the condition $|\mathbb{W}|>0$ requires a peculiar relationship between $\beta$ and the initial datum $f_{0}$.

First we state a generalization of Theorem 1 from Ref. [14] stating the global existence in the case when $|\mathbb{W}|>0$.

Theorem 3. Let Assumption 1 be satisfied. For any probability density $f_{0}$ in $L^{\infty}(\mathbb{V})$ such that $|\mathbb{W}|>0$, there exists for any $T>0$ a unique solution of Eq. (3) in $C^{1}\left(0, T ; L^{\infty}(\mathbb{V})\right)$. Moreover, $f=f(t)$ is a probability density for each $t>0$.

Proof. Uniqueness, positivity and conservation of the $L^{1}$-norm were already shown (a priori) in Ref. [17].

We have Eq. (14). Note that for $w, \widetilde{w} \in \mathbb{W}_{\beta}$ and $v \in \mathbb{V}$ we have $\beta(w, v)=\beta(\widetilde{w}, v)$. It follows $z(t, w)=z(t, \widetilde{w})$ and $u(t, w)=u(t, \widetilde{w})$. Then for $w, \widetilde{w} \in \mathbb{W}_{0}$, by $f_{0}(w)=f_{0}(\widetilde{w})$, we obtain $f(t, w)=f(t, \widetilde{w})$. By the non-negativity of the solution we have

$$
1=\int_{\mathbb{V}} f(t, v) \mathrm{d} v \geq \int_{\mathbb{W}_{0}} f(t, v) \mathrm{d} v=f(t, w)\left|\mathbb{W}_{0}\right|, \quad w \in \mathbb{W}_{0}
$$

Since $\left|\mathbb{W}_{0}\right| \geq|\mathbb{W}|>0$ we deduce an upper bound of the solution on the subset $\mathbb{W}$, and therefore the global existence. 
Now we state blow-up properties in a way similar to the respective part of Theorem 2 in Ref. [14] dedicated to blow-ups for the constant $\beta$ case.

We consider $\beta$ that is factorized, i.e.

$$
\beta(v, w)=\beta_{1}(v) \beta_{1}(w), \quad \text { for } \quad v, w \in \mathbb{V},
$$

with some function $\beta_{1}$.

Denote

$$
\begin{gathered}
\Phi(u, v)=\left(\int_{\mathbb{V}} \frac{f_{0}(w)}{\left(1-(\gamma-1) f_{0}^{\gamma-1}(w) \frac{\beta_{1}(w)}{\beta_{1}(v)} u\right)^{\frac{1}{\gamma-1}}} \mathrm{~d} w\right)^{1-\gamma}, \\
u_{0}(v)=\frac{\beta_{1}(v)}{(\gamma-1)\left\|f_{0}^{\gamma-1} \beta_{1}\right\|_{\infty}},
\end{gathered}
$$

and

$$
\Phi\left(u_{0}, v\right)=\lim _{u \uparrow u_{0}(v)} \Phi(u, v) .
$$

If we replace the integral over $\mathbb{V}$ by the integral over $\mathbb{W}_{\beta}$ we have the respective definition of $\Phi_{\beta}(u, v)$.

In order to overcome technical difficulties in the case $|\mathbb{W}|=0$, we precede our blow-up and global existence results by the following useful lemma:

Lemma 4. Let Assumption 1 together with Eq. (15) be satisfied, the probability density $f_{0}$ be in $L^{\infty}(\mathbb{V})$ and $|\mathbb{W}|=0$ but $\mathbb{W} \neq \emptyset$. Then any solution $f$ to Eq. (3) a priori satisfies

1. For all $v \in \mathbb{V}$

$$
\left(\beta_{-}-\frac{1}{\beta_{-}}\right)\left(\beta * f^{\gamma}\right)(t, v) \leq \frac{\left(\beta * \partial_{t} f\right)(t, v)}{(\beta * f)(t, v)} \leq\left(\frac{1}{\beta_{-}}-\beta_{-}\right)\left(\beta * f^{\gamma}\right)(t, v)
$$

2. $\beta_{-} z(t, v) \leq z(t, w) \leq \frac{1}{\beta_{-}} z(t, v)$ for all $v, w \in \mathbb{V}$, hence there are $c_{1}>0$ and $c_{2}>0$ such that

$$
c_{1}\left(\partial_{t} u(t, v)\right)^{\frac{1}{\beta_{-}(\gamma-1)}} \leq e^{-z(t, w)} \leq c_{2}\left(\partial_{t} u(t, v)\right)^{\frac{\beta_{-}}{\gamma-1}}
$$

3. For each $c_{0}>0$ there exists $c_{1}>0$ such that

$$
\begin{aligned}
& \left(\beta * f_{0}\right)^{-\frac{\beta_{-}}{\gamma-1}}+c_{0} \int_{\mathbb{V}} f_{0}(w)\left(\frac{1}{\left(1-(\gamma-1) f_{0}^{\gamma-1}(w) u(t, w)\right)^{\frac{1}{\gamma-1}}}-1\right) \mathrm{d} w \leq \\
& \leq c_{1} \int_{\mathbb{V}} \frac{f_{0}(w)}{\left(1-(\gamma-1) f_{0}^{\gamma-1}(w) u(t, w)\right)^{\frac{1}{\gamma-1}}} \mathrm{~d} w ;
\end{aligned}
$$


4. If $\int_{\mathbb{W}_{\beta}} f_{0} d w>0$, then for each $c_{0}>0$ there exists $c_{1}>0$ such that

$$
\begin{aligned}
& c_{1} \int_{\mathbb{W}_{\beta}} \frac{f_{0}(w)}{\left(1-(\gamma-1) f_{0}^{\gamma-1}(w) u(t, w)\right)^{\frac{1}{\gamma-1}}} \mathrm{~d} w \leq \\
& \leq\left(\beta * f_{0}\right)^{-\frac{1}{\beta_{-}(\gamma-1)}}+c_{0} \int_{\mathbb{W}_{\beta}} f_{0}(w)\left(\frac{1}{\left(1-(\gamma-1) f_{0}^{\gamma-1}(w) u(t, w)\right)^{\frac{1}{\gamma-1}}}-1\right) \mathrm{d} w .
\end{aligned}
$$

Proof. By Eqs (3) and (5) we obtain

$$
\begin{aligned}
& \left(\beta * \partial_{t} f\right)(t, v)=\left(\beta *\left(f^{\gamma}(\beta * f)-f\left(\beta * f^{\gamma}\right)\right)\right)(t, v)= \\
& =\int_{\mathbb{V}} \beta(v, w)\left(f^{\gamma}(t, w) \int_{\mathbb{V}} \beta(w, \bar{w}) f(t, \bar{w}) \mathrm{d} \bar{w}\right. \\
& \left.-f(t, w) \int_{\mathbb{V}} \beta(w, \bar{w}) f^{\gamma}(t, \bar{w}) \mathrm{d} \bar{w}\right) \mathrm{d} w \leq \\
& \leq \frac{1}{\beta_{-}} \int_{\mathbb{V}} \beta(v, w) f^{\gamma}(t, w) \int_{\mathbb{V}} \beta(v, \bar{w}) f(t, \bar{w}) \mathrm{d} \bar{w} d w \\
& -\beta_{-} \int_{\mathbb{V}} \beta(v, \bar{w}) f^{\gamma}(t, \bar{w}) \int_{\mathbb{V}} \beta(v, w) f(t, w) \mathrm{d} w \mathrm{~d} \bar{w}= \\
& =\left(\frac{1}{\beta_{-}}-\beta_{-}\right)\left(\beta * f^{\gamma}\right)(t, v)(\beta * f)(t, v) .
\end{aligned}
$$

Hence the upper innequality in the assertion (1) is proved. The lower inequality can be proved in a similar way.

To prove (2) we start from the observation that, by Eq. (5) we have $\beta_{-}\left(\beta * f^{\gamma}\right)(t, v) \leq\left(\beta * f^{\gamma}\right)(t, w) \leq \frac{1}{\beta_{-}}\left(\beta * f^{\gamma}\right)(t, v)$. Thus $\beta_{-} z(t, v) \leq$ $z(t, w) \leq \frac{1}{\beta_{-}} z(t, v)$. From (13) we get

$$
e^{-z(t, v)}=\frac{\left(\partial_{t} u(t, v)\right)^{\frac{1}{\gamma-1}}}{((\beta * f)(t, v))^{\frac{1}{\gamma-1}}}
$$

Applying Assumption 1, we have the uniform estimates $\beta_{-} \beta_{+} \leq \beta * f \leq \beta_{+}$ for any probability density $f$. Hence there exists a positive constant $c_{2}$ such that

$$
e^{-z(t, w)} \leq e^{-\beta_{-} z(t, v)}=\frac{\left(\partial_{t} u(t, v)\right)^{\frac{\beta_{-}}{\gamma-1}}}{((\beta * f)(t, v))^{\frac{\beta_{-}}{\gamma-1}}} \leq c_{2}\left(\partial_{t} u(t, v)\right)^{\frac{\beta_{-}}{\gamma-1}} .
$$

By the same arguments we get

$$
e^{-z(t, w)} \geq e^{-\frac{1}{\beta_{-}} z(t, v)} \geq c_{1}\left(\partial_{t} u(t, v)\right)^{\frac{1}{\beta_{-}(\gamma-1)}}
$$

with some positive constant $c_{1}$. 
We prove the assertion (3). Let $c_{0}>0$. Since $\beta * f_{0}$ is bounded and separated from zero, there exists a constant $c_{1}>0$ such that $c_{1} \geq c_{0}$ and

$$
c_{1} \geq\left(\beta * f_{0}\right)^{-\frac{\beta_{-}}{\gamma-1}} .
$$

With this constant we are ready to demonstrate the inequality of the assertion (3) as follows:

$$
\begin{aligned}
& \left(\beta * f_{0}\right)^{-\frac{\beta_{-}}{\gamma-1}}+c_{0} \int_{\mathbb{V}} f_{0}(w)\left(\frac{1}{\left(1-(\gamma-1) f_{0}^{\gamma-1}(w) u(t, w)\right)^{\frac{1}{\gamma-1}}}-1\right) \mathrm{d} w \leq \\
& \leq c_{1}+c_{1} \int_{\mathbb{V}} f_{0}(w)\left(\frac{1}{\left(1-(\gamma-1) f_{0}^{\gamma-1}(w) u(t, w)\right)^{\frac{1}{\gamma-1}}}-1\right) \mathrm{d} w= \\
& =c_{1} \int_{\mathbb{V}} \frac{f_{0}(w)}{\left(1-(\gamma-1) f_{0}^{\gamma-1}(w) u(t, w)\right)^{\frac{1}{\gamma-1}}} \mathrm{~d} w,
\end{aligned}
$$

where we have used the identity $\int_{\mathbb{V}} f_{0} \mathrm{~d} w=1$.

We prove the assertion (4). Let $c_{0}>0$. Then there exists a positive constant $c_{1}$ such that

$$
c_{1} \leq c_{0} \int_{\mathbb{W}_{\beta}} f_{0}(w) \mathrm{d} w, \quad c_{1} \leq\left(\beta * f_{0}\right)^{-\frac{1}{\beta_{-}(\gamma-1)}} .
$$

It is easy to see that the assertion (4) is satisfied.

Theorem 5. Let Assumption 1 together with Eq. (15) be satisfied, the probability density $f_{0}$ be in $L^{\infty}(\mathbb{V})$ and $|\mathbb{W}|=0$ but $\mathbb{W} \neq \emptyset$. Additionally, let $f_{0}$ and $\beta$ be continuous functions, $v \in \mathbb{W}, u_{0}=u_{0}(v)$ and one of the following conditions be satisfied

1. $\Phi\left(u_{0}, v\right)>0$,

2. $\Phi\left(u_{0}, v\right)=0$ and

$$
\int_{0}^{u_{0}} \frac{1}{(\Phi(u, v))^{\beta_{-}}} \mathrm{d} u<\infty .
$$

Then there is a blow-up in a finite time $T>0$. This blow-up occurs for all $v \in \mathbb{W}$.

Proof. It is easy to see that if $f_{0}$ and $\beta$ are continuous functions then the corresponding solution is a prori continuous. Let $v \in \mathbb{V}$. Differentiating Eq. (13) and applying Eq. (11), we have

$$
\frac{\partial_{t t} u(t, v)}{\partial_{t} u(t, v)}=\frac{\left(\beta * \partial_{t} f\right)(t, v)}{(\beta * f)(t, v)}-(\gamma-1)\left(\beta * f^{\gamma}\right)(t, v) .
$$


Applying Lemma 4,(1) we arrive at the inequality

$$
\frac{\partial_{t t} u(t, v)}{\partial_{t} u(t, v)} \geq-\mathfrak{c}\left(\beta * f^{\gamma}\right)(t, v),
$$

where $\mathfrak{c}$ is reserved for positive constants (if $\mathfrak{c}$ appears in a formula it means that the formula is valid with some positive $\mathfrak{c}$ ). Taking into account Eq. (12) and Lemma 4,(2) we have

$$
\begin{aligned}
\frac{\partial_{t t} u(t, v)}{\partial_{t} u(t, v)} \geq-\mathfrak{c} \int_{\mathbb{V}} f_{0}(w) \frac{(\gamma-1) f_{0}^{\gamma-1}(w) \partial_{t} u(t, w) e^{-z(t, w)}}{\left(1-(\gamma-1) f_{0}^{\gamma-1}(w) u(t, w)\right)^{\frac{\gamma}{\gamma-1}}} \mathrm{~d} w \geq \\
\geq-\mathfrak{c} \int_{\mathbb{V}} f_{0}(w) \frac{(\gamma-1) f_{0}^{\gamma-1}(w) \partial_{t} u(t, w)\left(\partial_{t} u(t, v)\right)^{\frac{\beta_{-}}{\gamma-1}}}{\left(1-(\gamma-1) f_{0}^{\gamma-1}(w) u(t, w)\right)^{\frac{\gamma}{\gamma-1}}} \mathrm{~d} w= \\
=-\mathfrak{c}\left(\partial_{t} u(t, v)\right)^{\frac{\beta_{-}}{\gamma-1}} \int_{\mathbb{V}} f_{0}(w) \partial_{t}\left(\frac{1}{\left(1-(\gamma-1) f_{0}^{\gamma-1}(w) u(t, w)\right)^{\frac{1}{\gamma-1}}}\right) \mathrm{d} w .
\end{aligned}
$$

Therefore

$$
\begin{aligned}
& \partial_{t} u(t, v) \geq\left(\left(\beta * f_{0}\right)^{-\frac{\beta_{-}}{\gamma-1}}+\right. \\
& \left.\mathfrak{c} \int_{\mathbb{V}} f_{0}(w)\left(\frac{1}{\left(1-(\gamma-1) f_{0}^{\gamma-1}(w) u(t, w)\right)^{\frac{1}{\gamma-1}}}-1\right) \mathrm{d} w\right)^{-\beta_{-}(\gamma-1)} .
\end{aligned}
$$

Now let $v \in \mathbb{W}$. By virtue of Lemma 4,(3) we may choose another positive constant (again denoted by $\mathfrak{c}$ ) such that

$$
\partial_{t} u(t, v) \geq \mathfrak{c}\left(\int_{\mathbb{V}} \frac{f_{0}(w)}{\left(1-(\gamma-1) f_{0}^{\gamma-1}(w) u(t, w)\right)^{\frac{1}{\gamma-1}}} \mathrm{~d} w\right)^{-\beta_{-}(\gamma-1)} .
$$

By Eq. (15) and the monotonicity (cf. the definition of $\mathbb{W}$ ) we obtain

$$
u(t, w) \leq \frac{\beta_{1}(w)}{\beta_{1}(v)} u(t, v) \quad \text { for } w \in \mathbb{V}, v \in \mathbb{W},
$$

and

$$
\partial_{t} u(t, v) \geq \mathfrak{c}\left(\int_{\mathbb{V}} \frac{f_{0}(w)}{\left(1-(\gamma-1) f_{0}^{\gamma-1}(w) \frac{\beta_{1}(w)}{\beta_{1}(v)} u(t, v)\right)^{\frac{1}{\gamma-1}}} \mathrm{~d} w\right)^{-\beta_{-}(\gamma-1)},
$$

i.e.

$$
\partial_{t} u(t, v) \geq \mathfrak{c}(\Phi(u(t, v), v))^{\beta_{-}} .
$$


For any $v \in \mathbb{W}$ we define the comparison equation

$$
\mathrm{d}_{t} U=\mathfrak{c}(\Phi(U, v))^{\beta_{-}},
$$

Note that the right-hand side is independent of the choice of $v \in \mathbb{W}$. There are two possibilities of blow-up: either the positive stationary solution $u_{0}$ admits the positive integral, or it is infinite and Eq. (16) holds. This ends the proof.

Remark 4. The assumption on the continuity of the initial datum and the function $\beta$ is quite restrictive and probably unnatural one. We may note that the statement is more general and may be relaxed, e.g. for the càdlàg functions in one-dimensional case $d=1$.

We propose now a generalization of Theorem 2 in Ref. [14] stating the global existence in the case when $|\mathbb{W}|=0$.

Theorem 6. Let

$$
\gamma-1-\frac{1}{\beta_{-}}+\beta_{-}>0
$$

Assumption 1 together with Eq. (15) be satisfied, a probability density $f_{0}$ be in $L^{\infty}(\mathbb{V}),|\mathbb{W}|=0$ but $\mathbb{W} \neq \emptyset$, and $\int_{\mathbb{W}_{\beta}} f_{0} \mathrm{~d} w>0$. Moreover, let $f_{0}$ and $\beta$ be continuous functions. If $\Phi_{\beta}\left(u_{0}, v\right)=0, v \in \mathbb{W}$, and

$$
\int_{0}^{u_{0}} \frac{1}{\left(\Phi_{\beta}(u, v)\right)^{\frac{1}{\beta_{-}}}} \mathrm{d} u=\infty .
$$

then, for any $T>0$, there exists a unique solution $f=f(t)$ of Eq. (3) in $C^{1}\left(0, T ; L^{\infty}(\mathbb{V})\right)$. Moreover, the solution $f(t)$ is a probability density and is a continuous function for $t \geq 0$.

Proof. Note that the "worst" situation in Eq. (12) may happen for $v \in \mathbb{W}$. Therefore we are going to check the possibility of estimates in this case. Differentiating Eq. (13) and applying Eq. (11), we have (17). Applying Lemma 4,(1) we obtain

$$
\begin{aligned}
& \frac{\partial_{t t} u(t, v)}{\partial_{t} u(t, v)}=\frac{\left(\beta * \partial_{t} f\right)(t, v)}{(\beta * f)(t, v)}-(\gamma-1)\left(\beta * f^{\gamma}\right)(t, v) \leq \\
& \leq-\left(\gamma-1-\frac{1}{\beta_{-}}+\beta_{-}\right)\left(\beta * f^{\gamma}\right)(t, v) \\
& =-\left(\gamma-1-\frac{1}{\beta_{-}}+\beta_{-}\right) \int_{\mathbb{V}} \frac{\beta(v, w) f_{0}^{\gamma}(w) e^{-\gamma z(t, w)}}{\left(1-(\gamma-1) f_{0}^{\gamma-1}(w) u(t, w)\right)^{\frac{\gamma}{\gamma-1}}} \mathrm{~d} w .
\end{aligned}
$$

Since $\left|\mathbb{W}_{\beta}\right|>0$ and $v \in \mathbb{W}$ we have

$$
\frac{\partial_{t t} u(t, v)}{\partial_{t} u(t, v)} \leq-\mathfrak{c} \int_{\mathbb{W}_{\beta}} \frac{f_{0}^{\gamma}(w) e^{-(\gamma-1) z(t, w)} e^{-z(t, w)}}{\left(1-(\gamma-1) f_{0}^{\gamma-1}(w) u(t, w)\right)^{\frac{\gamma}{\gamma-1}}} \mathrm{~d} w,
$$


where here and subsequently by symbol $\mathfrak{c}$ we denote a (generic) positive constant. By Lemma 4,(2) we have

$$
e^{-(\gamma-1) z(t, w)} e^{-z(t, w)}=\frac{\partial_{t} u(t, w)}{\beta * f(t, w)} e^{-z(t, w)} \geq \mathfrak{c} \partial_{t} u(t, w)\left(\partial_{t} u(t, v)\right)^{\frac{1}{\beta_{-}(\gamma-1)}}
$$

Therefore for $v \in \mathbb{W}$

$$
\begin{aligned}
& \frac{\partial_{t t} u(t, v)}{\partial_{t} u(t, v)}\left(\partial_{t} u(t, v)\right)^{-\frac{1}{\beta_{-}(\gamma-1)}} \leq \\
& \leq-\mathfrak{c} \int_{\mathbb{W}_{\beta}} f_{0}(w) \frac{(\gamma-1) f_{0}^{\gamma-1}(w) \partial_{t} u(t, w)}{\left(1-(\gamma-1) f_{0}^{\gamma-1}(w) u(t, w)\right)^{\frac{\gamma}{\gamma-1}}} \mathrm{~d} w \\
& =-\mathfrak{c} \int_{\mathbb{W}_{\beta}} f_{0}(w) \partial_{t}\left(\frac{1}{\left(1-(\gamma-1) f_{0}^{\gamma-1}(w) u(t, w)\right)^{\frac{1}{\gamma-1}}}\right) \mathrm{d} w .
\end{aligned}
$$

Integrating yields

$$
\begin{aligned}
& \left(\partial_{t} u(t, v)\right)^{-\frac{1}{\beta_{-}(\gamma-1)}} \geq\left(\beta * f_{0}\right)^{-\frac{1}{\beta_{-}(\gamma-1)}}(v)+ \\
& +\mathfrak{c} \int_{\mathbb{W}_{\beta}} f_{0}(w)\left(\frac{1}{\left(1-(\gamma-1) f_{0}^{\gamma-1}(w) u(t, w)\right)^{\frac{1}{\gamma-1}}}-1\right) \mathrm{d} w
\end{aligned}
$$

and, recalling Lemma 4,(4), we get

$$
\begin{aligned}
& \partial_{t} u(t, v) \leq\left(\left(\beta * f_{0}\right)^{-\frac{1}{\beta_{-}(\gamma-1)}}(v)+\right. \\
& \left.+\mathfrak{c} \int_{\mathbb{W}_{\beta}} f_{0}(w)\left(\frac{1}{\left(1-(\gamma-1) f_{0}^{\gamma-1}(w) \frac{\beta_{1}(w)}{\beta_{1}(v)} u(t, v)\right)^{\frac{1}{\gamma-1}}}-1\right) \mathrm{d} w\right)^{-\frac{1}{\beta_{-}(\gamma-1)}} \\
& \leq \mathfrak{c}\left(\int_{\mathbb{W}_{\beta}} \frac{f_{0}(w)}{\left(1-(\gamma-1) f_{0}^{\gamma-1}(w) \frac{\beta_{1}(w)}{\beta_{1}(v)} u(t, v)\right)^{\frac{1}{\gamma-1}}} \mathrm{~d} w\right)^{-\frac{1}{\beta_{-}(\gamma-1)}} \\
& =\mathfrak{c}\left(\Phi_{\beta}(u(t, v), v)\right)^{\frac{1}{\beta_{-}}} .
\end{aligned}
$$

Now we may define the comparison equation

$$
\mathrm{d}_{t} U(t, v)=\mathfrak{c}\left(\Phi_{\beta}(U, v)\right)^{\frac{1}{\beta_{-}}} .
$$

The right-hand side does not depend on the choice of $v \in \mathbb{W}$. The assumption $\Phi_{\beta}\left(u_{0}, v\right)=0$ and (18) implies global existence of $U$, hence $u(t, v)$ exists globally. 
Remark 5. The similar remark as Remark 4 may be related to Theorem 6 .

Remark 6. We may consider a simplification of the operator $Q_{N}$ in Eq. (2). Denote by $(\beta * f)$ the convolution-like product in $\Omega \times \mathbb{V}$, i.e.

$$
(\beta * f)(t, v)=\int_{\mathbb{V}} \int_{\Omega} \beta(v, w) f(t, y, w) \mathrm{d} y \mathrm{~d} w,
$$

that however does not depend on $x$.

We can apply the technique developed in this sections to the following simplified version of Eq. (2)

$$
\begin{aligned}
& \partial_{t} f(t, x, v)+v \cdot \nabla_{x} f(t, x, v)= \\
& =(\beta * f)(t, v) f^{\gamma}(t, x, v)-\left(\beta * f^{\gamma}\right)(t, v) f(t, x, v)
\end{aligned}
$$

with the initial data $f(0, x, v)=f_{0}(x, v)$ that is a probability density on $\Omega \times \mathbb{V}$ and $\Omega=\mathbb{R}^{d}$.

We study this equation along characteristics (see Remark 3)

$$
\begin{aligned}
& \partial_{t} f^{\#}(t, x, v)=(\beta * f)(t, v)\left(f^{\gamma}\right)^{\#}(t, x, v) \\
& -f^{\#}(t, x, v)\left(\beta * f^{\gamma}\right)(t, v) .
\end{aligned}
$$

Integrating yields

$$
f^{\#}(t, x, v)=\frac{f_{0}(x, v) e^{-z(t, v)}}{\left(1-(\gamma-1) f_{0}(x, v) u(t, v)\right)^{\frac{1}{\gamma-1}}},
$$

where

$$
z(t, v)=\int_{0}^{t}\left(\beta * f^{\gamma}\right)(s, v) \mathrm{d} s
$$

and

$$
u(t, v)=\int_{0}^{t}(\beta * f)(s, v) \exp (-(\gamma-1) z(s, v)) \mathrm{d} s .
$$

It is easy to see that that we may formulate the theorems analogous to theorems of this section in this case.

\subsection{Analysis in $L^{\gamma}(\mathbb{V})$ setting}

In this section we are going to discuss the question of blow-up or global boundedness (on compact time intervals) with respect to $L^{p}$-norms, $1 \leq$ $p<\infty$. It is clear that global existence in $L^{\infty}$ implies the boundedness (on compact time intervals) of any $L^{p}$-norm for $p \geq 1$. On the other hand it is 
not obvious whether blow-ups studied in previous section lead to blow-ups in terms of $L^{\gamma}$-norm.

We are going to express blow-up and global boundedness in $L^{\gamma}$ in terms of the following quantities

$$
M_{p, 0}=\int_{\mathbb{V}} f_{0}^{p}(v) \mathrm{d} v, \quad M_{p}(t)=\int_{\mathbb{V}} f^{p}(t, v) \mathrm{d} v,
$$

referred to the initial datum $f_{0}$ and the corresponding solution $f=f(t)$, respectively. We have

$$
\begin{aligned}
& \mathrm{d}_{t} M_{p}(t)=p \int_{\mathbb{V}} f^{p-1}(t, v) \mathrm{d}_{t} f(t, v) \mathrm{d} v \\
& =\frac{p}{2}\left(\int_{\mathbb{V}} f^{p-1+\gamma}(t, v) \int_{\mathbb{V}} \beta(v, w) f(t, w) \mathrm{d} w \mathrm{~d} v+\right. \\
& +\int_{\mathbb{V}} f^{p-1+\gamma}(t, w) \int_{\mathbb{V}} \beta(v, w) f(t, v) \mathrm{d} v \mathrm{~d} w \\
& -\int_{\mathbb{V}} f^{p}(t, v) \int_{\mathbb{V}} \beta(v, w) f^{\gamma}(t, w) \mathrm{d} w \mathrm{~d} v \\
& \left.-\int_{\mathbb{V}} f^{p}(t, w) \int_{\mathbb{V}} \beta(v, w) f^{\gamma}(t, v) \mathrm{d} v \mathrm{~d} w \cdot\right)
\end{aligned}
$$

Therefore

$$
\begin{aligned}
& \mathrm{d}_{t} M_{p}(t)=\frac{p}{2} \int_{\mathbb{V}^{2}} \beta(v, w)\left(f^{p+\gamma-1}(t, v) f(t, w)+f^{p+\gamma-1}(t, w) f(t, v)\right. \\
& \left.-f^{p}(t, v) f^{\gamma}(t, w)-f^{p}(t, w) f^{\gamma}(t, v)\right) \mathrm{d} v \mathrm{~d} w
\end{aligned}
$$

and

$$
\begin{aligned}
& \mathrm{d}_{t} M_{p}(t)=\frac{p}{2} \int_{\mathbb{V}^{2}} \beta(w, v) f(t, w) f(t, v)\left(f^{p-1}(t, w)-f^{p-1}(t, v)\right) \times \\
& \times\left(f^{\gamma-1}(t, w)-f^{\gamma-1}(t, v)\right) \mathrm{d} w \mathrm{~d} v .
\end{aligned}
$$

It follows that each quantity $M_{p}, p>1$, is increasing. Let

$$
H_{\lambda}(r)=1+\sum_{k=1}^{\infty} \gamma(2 \gamma-1) \ldots(k \gamma-k+1) M_{k \gamma-k+1,0} \frac{\lambda^{k} r^{k}}{k !} .
$$

We note that the series (20) is convergent for small $\lambda r$ : Its radius of convergence can be calculated by the d'Alembert criterion. We have

$$
\lim _{k \rightarrow \infty} \frac{((k+1) \gamma-k) M_{(k+1) \gamma-k, 0}}{(k+1) M_{k \gamma-k+1,0}} \lambda r=(\gamma-1) \lambda r\left\|f_{0}\right\|_{\infty}^{\gamma-1}
$$


and hence

$$
r<r_{0}=\frac{1}{(\gamma-1) \lambda\left\|f_{0}\right\|_{\infty}^{\gamma-1}}
$$

in order to guarantee the convergence.

The following criterion of global boundedness does not require a separation of $\beta$ from 0 .

Theorem 7 (Global boundedness in $L^{\gamma}$ ). Let the probability density $f_{0}$ be in $L^{\infty}$ and $\beta \in L^{\infty}\left(\mathbb{V}^{2}\right)$. Additionally let the initial value problem

$$
\mathrm{d}_{t} Y=\frac{1}{\left(H_{\|\beta\|_{\infty}}(Y)\right)^{\gamma-1}}, \quad Y(0)=0,
$$

has a global solution. Then the solution $f=f(t)$ of Eq. (3) is a priori bounded in $L^{\gamma}$ on each compact time interval.

Proof. We first observe that the assumptions of Theorem 7 imply that the solution $Y=Y(t)$ to Eq. (22) satisfies

$$
Y(t)<\frac{1}{(\gamma-1)\|\beta\|_{\infty}\left\|f_{0}\right\|_{\infty}^{\gamma-1}} \quad \forall t \in(0, \infty),
$$

where the number on the RHS of Inequality (23) is the radius of convergence of the series (20) for $\lambda=\|\beta\|_{\infty}$ - cf. Eq. (21).

By Eq. (19) we obtain

$$
\mathrm{d}_{t} M_{p} \leq p\|\beta\|_{\infty}\left(M_{p+\gamma-1}-M_{p} M_{\gamma}\right) .
$$

Hence we have an inequality

$$
\begin{aligned}
& \mathrm{d}_{t}\left(M_{p}(t) \exp \left(p\|\beta\|_{\infty} \int_{0}^{t} M_{\gamma}(s) \mathrm{d} s\right)\right) \leq \\
& \leq p\|\beta\|_{\infty} M_{p+\gamma-1}(t) \exp \left(p\|\beta\|_{\infty} \int_{0}^{t} M_{\gamma}(s) \mathrm{d} s\right),
\end{aligned}
$$

or denoting

$$
\widetilde{M}_{p}(t)=M_{p}(t) \exp \left(p\|\beta\|_{\infty} \int_{0}^{t} M_{\gamma}(s) \mathrm{d} s\right)
$$

and

$$
Q=Q(t)=\exp \left((\gamma-1)\|\beta\|_{\infty} \int_{0}^{t} M_{\gamma} \mathrm{d} \tau\right)
$$

we have

$$
\widetilde{M}_{p}(t) \leq M_{p, 0}+p\|\beta\|_{\infty} \int_{0}^{t} \frac{\widetilde{M}_{p+\gamma-1}(s)}{Q(s)} \mathrm{d} s .
$$


Iterating yields

$$
\begin{aligned}
& \widetilde{M}_{\gamma}(t) \leq M_{\gamma, 0}+\sum_{k=1}^{\infty} \gamma(2 \gamma-1) \ldots(k \gamma-k+1)\|\beta\|_{\infty}^{k} M_{k \gamma-k+1,0} \times \\
& \times \int_{0}^{t} \frac{1}{Q\left(t_{1}\right)} \int_{0}^{t_{1}} \frac{1}{Q\left(t_{2}\right)} \cdots \int_{0}^{t_{k-1}} \frac{1}{Q\left(t_{k}\right)} \mathrm{d} t_{k} \ldots \mathrm{d} t_{1},
\end{aligned}
$$

and

$$
\begin{aligned}
& M_{\gamma}(t) \exp \left(\|\beta\|_{\infty} \int_{0}^{t} M_{\gamma}(\tau) \mathrm{d} \tau\right) \leq \frac{M_{\gamma, 0}}{Q(t)}+\sum_{k=1}^{\infty} \gamma(2 \gamma-1) \ldots(k \gamma-k+1) \times \\
& \times\|\beta\|_{\infty}^{k} \frac{M_{k \gamma-k+1,0}}{Q(t)} \int_{0}^{t} \frac{1}{Q\left(t_{1}\right)} \int_{0}^{t_{1}} \frac{1}{Q\left(t_{2}\right)} \cdots \int_{0}^{t_{k-1}} \frac{1}{Q\left(t_{k}\right)} \mathrm{d} t_{k} \ldots \mathrm{d} t_{1} .
\end{aligned}
$$

Integrating on $[0, t]$ yields

$$
\begin{aligned}
& \frac{Q^{\frac{1}{\gamma-1}}(t)-1}{\|\beta\|_{\infty}} \leq \int_{0}^{t} \frac{M_{\gamma, 0}}{Q\left(t_{1}\right)}+\sum_{k=1}^{\infty} \gamma(2 \gamma-1) \ldots(k \gamma-k+1) \times \\
& \times\|\beta\|_{\infty}^{k} \frac{M_{k \gamma-k+1,0}}{Q(t)} \int_{0}^{t_{1}} \frac{1}{Q\left(t_{2}\right)} \int_{0}^{t_{2}} \frac{1}{Q\left(t_{3}\right)} \ldots \int_{0}^{t_{k}} \frac{1}{Q\left(t_{k+1}\right)} \mathrm{d} t_{k+1} \ldots \mathrm{d} t_{1} .
\end{aligned}
$$

that is

$$
Q(t) \leq\left(H_{\|\beta\|_{\infty}}\left(\int_{0}^{t} \frac{1}{Q(s)} \mathrm{d} s\right)\right)^{\gamma-1} .
$$

As we observed earlier the quantities $M_{p}$ are increasing for $p>1$, hence $Q$ is an increasing function as well. Among the increasing functions that satisfy Inequality (24) the largest is a function for which the inequality becomes the equality. Thus we can claim that

$$
Q(t) \leq \frac{1}{\mathrm{~d}_{t} Y(t)}=\left(H_{\|\beta\|_{\infty}}(Y(t))\right)^{\gamma-1}, \quad t>0
$$

where $Y$ is the solution to Eq. (22).

Keeping in mind that $Y$ is strictly increasing and Eq. (23) is satisfied we conclude that $Q(t)$ is bounded on any compact time interval and hence $M_{\gamma}(t)=\|f(t, .)\|_{\gamma}^{\gamma}$ is also bounded on any compact time interval. This ends the proof.

Theorem 8 (Blow-up of the $L^{\gamma}$-norm). Let the probability density $f_{0}$ be in $L^{\infty}$ and Assumption 1 be satisfied. Additionally let the IVP

$$
\mathrm{d}_{t} Y=\frac{1}{\left(H_{\underline{\beta}}(Y)\right)^{\gamma-1}}, \quad Y(0)=0, \quad \underline{\beta}=\beta_{-} \beta_{+},
$$

have a solution only for $t \in[0, T)$. Then the $L^{\gamma}$-norm of the solution $f=$ $f(t)$ of Eq. (3) blows up before $T$. 
Proof. We first observe that the assumptions of Theorem 8 imply that the solution $Y=Y(t)$ to Eq. (25) satisfies

$$
Y(t)<\frac{1}{(\gamma-1) \underline{\beta}\left\|f_{0}\right\|_{\infty}^{\gamma-1}}, \quad \forall t \in[0, T),
$$

and

$$
\lim _{t \rightarrow T} Y(t)=\frac{1}{(\gamma-1) \underline{\beta}\left\|f_{0}\right\|_{\infty}^{\gamma-1}} .
$$

By Eq. (19) we obtain

$$
\mathrm{d}_{t} M_{p} \geq p \underline{\beta}\left(M_{p+\gamma-1}-M_{p} M_{\gamma}\right),
$$

for $p \geq \gamma$. By iteration we obtain the desired lower estimate in terms of $Y(t)$, that is

$$
Q(t) \geq\left(H_{\underline{\beta}}\left(\int_{0}^{t} \frac{1}{Q(s)} \mathrm{d} s\right)\right)^{\gamma-1}
$$

and hereafter

$$
Q(t) \geq \frac{1}{\mathrm{~d}_{t} Y(t)}=\left(H_{\underline{\beta}}(Y(t))\right)^{\gamma-1}, \quad t>0,
$$

where $Y$ is the solution to Eq. (25).

Keeping in mind that $Y$ is strictly increasing and Eqs. (26) and (27) are satisfied we obtain that $Q(t)$ is bounded on a subinterval of $[0, T)$. This ends the proof.

Corollary 8.1. Let the probability density $f_{0}$ be in $L^{\infty}$ and $\beta \in L^{\infty}\left(\mathbb{V}^{2}\right)$ (not necessarily separated below from zero) and

$$
\int_{0}^{\frac{u_{0}}{\|\beta\| \infty}}\left(H_{\|\beta\|_{\infty}}(y)\right)^{\gamma-1} \mathrm{~d} y=+\infty .
$$

Then the solution $f=f(t)$ of Eq. (3) is a priori bounded in $L^{\gamma}$ on each compact time interval.

If $f_{0}$ belongs to $L^{\infty}$ and $\beta \in L^{\infty}\left(\mathbb{V}^{2}\right)$ is separated from zero $\beta \geq \underline{\beta}>0$ and

$$
\int_{0}^{\frac{u_{0}}{\beta}}\left(H_{\underline{\beta}}(y)\right)^{\gamma-1} \mathrm{~d} y<+\infty,
$$

then a blow-up of the $L^{\gamma}-$ norm occurs.

Remark 7. By the theory of ODE's the upper bound of the blow-up time T coincides with $\int_{0}^{\frac{u_{0}}{\beta}}\left(H_{\underline{\beta}}(y)\right)^{\gamma-1} \mathrm{~d} y$. 


\section{Concluding remarks - Challenging problems}

Ref. [14] and the present paper clearly show that Eq. (7) and Eq. (3) may generate very complex behavior of solutions. The constant $\beta$ (i.e. Eq. (7)) case studied in Ref. [14] leads to the full description of blow-up versus global existence behavior. The case of non-constant $\beta$, studied in the present paper, is certainly more complex and we are quite far from the full description for the general $\beta$. In the present paper we proved that the methods of Ref. [14] can be generalized in order to state similar results but under some restrictive assumptions on $\beta$ itself. A very interesting both from theoretical and applicative points of views case is $\beta$ that satisfies

$$
\beta(v, w)=\beta_{0}(|w-v|),
$$

where the function $\beta_{0}$ has a "small" support. The theory developed in the present paper - that bases on Assumption 1 or factorization (15) — does not cover case (28).

In numerous simulations we observe a tendency to blowing-up. For example consider $\mathbb{V}=[0,1], \gamma=2$,

$$
\beta_{0}(v)=\chi(v \leq r), \quad v \geq 0,
$$

where $\chi($ true $)=1, \chi($ false $)=0$ and $r=\frac{1}{4}$.

Then we have - see Figure ?? -

It turns out that the methods of Section 2.2, i.e. the criteria of $L^{\gamma}$ blowup and global existence, can be applied to Eq. (1) or Eq. (2). One can redefine the quantities $M_{p, 0}$ and $M_{p}$

$$
M_{p, 0}=\int_{\mathbb{R}^{d}} \int_{\mathbb{V}} f_{0}^{p}(x, v) \mathrm{d} v d x, \quad M_{p}(t)=\int_{\mathbb{R}^{d}} \int_{\mathbb{V}} f^{p}(t, x, v) \mathrm{d} v \mathrm{~d} x .
$$

and use

$$
\int_{\mathbb{R}^{d}} \nabla_{x} f^{p} \mathrm{~d} x=0 .
$$

This problem will be studied in a further publication.

\section{References}

[1] G. Ajmone Marsan, N. Bellomo, L. Gibelli, Stochastic evolutionary differential games toward a system theory of behavioral social dynamics, Math. Models Methods Appl. Sci 26 (2016) 1051-1093.

[2] L. Arlotti, N. Bellomo, E. De Angelis, M. Lachowicz, Generalized Kinetic Models in Applied Sciences, World Sci., Singapore 2003. 
[3] J. Banasiak, M. Lachowicz, On a macroscopic limit of a kinetic model of alignment, Math. Models Methods Appl. Sci., 23 (2013) 2647-2670.

[4] J. Banasiak, M. Lachowicz, Methods of small parameter in mathematical biology, Birkhäuser, Boston 2014.

[5] N. Bellomo, J. Soler, On the mathematical theory of the dynamics of swarms viewed as complex systems, Math. Models Methods Appl. Sci. 22 (2012) 1140006

[6] E. Carlen, P. Degond, B. Wennberg, Kinetic limits for pair-interaction driven master equation and biological swarm models, Math. Models Methods Appl. Sci. 23 (2013) 1339-1376.

[7] J.A. Carrillo, M.R. D'Orsogna, V. Panferov, Double milling in selfpropelled swarms from kinetic theory, Kinetic Related Models 2 (2009) 363-378.

[8] J. A. Carrillo, M. Fornasier, G. Toscani, F. Vecil, Particle, Kinetic, and Hydrodynamic Models of Swarming, Mathematical Modeling of Collective Behavior in Socio-Economic and Life Sciences, G. Naldi, L. Pareschi and G. Toscani Eds. Birkhäuser, Boston (2010) 297-336.

[9] P. Degond, A. Frouvelle, G. Raoul, Local stability of perfect alignment for a spatially homogeneous kinetic model, J. Stat. Phys. 157 (2014) 84-112.

[10] E. Geigant, M. Stoll, Bifurcation analysis of an orientational aggregation model, J. Math Biol. 46 (2003), 537-563.

[11] S. Kaniel, M. Shinbrot, The Boltzmann equation. I. Uniqueness and local existence, Commun. Math. Phys. 58 (1978) 65-84.

[12] K. Kang, B. Perthame, A. Stevens, J.J.L. Valázquez, An integrodifferential equation model for alignment and orientational aggregation, J. Diff. Eqs. 246 (2009), 1387-1421.

[13] M. Lachowicz, Individually-based Markov processes modeling nonlinear systems in mathematical biology, Nonlinear Analysis Real World Appl. 12 (2011) 2396-2407.

[14] M. Lachowicz, H. Leszczyński, M. Parisot, A simple kinetic equation of swarm formation: blow-up and global existence, Appl. Math. Letters 57 (2016) 104-107.

[15] H. Ninomiya, M. Fila, Reaction versus diffusion: blow-up induced and inhibited by diffusivity, Russian Math. Surveys 60 (2005) 1217-1235. 
[16] L. Pareschi and G. Toscani, Interacting Multiagent Systems: Kinetic equations and Monte Carlo methods, Oxford University Press (2013).

[17] M. Parisot, M. Lachowicz, A kinetic model for the formation of swarms with nonlinear interactions, Kinetic Related Models 9 (2016) 131-164.

[18] Special Issue: Collective Behavior, J. Statist. Phys. 158 (2015).

[19] P. Quittner, P. Souplet, Superlinear Parabolic Problems. Blow-up, Global Existence and Steady States. Birkhäuser Advanced Texts, Basel (2007).

[20] W. Walter, Ordinary Differential Equations, Springer, New York (1998). 